\title{
Motor Competence and Physical Activity in Early Childhood: Stability and Relationship
}

\begin{abstract}
Einat A. Schmutz ${ }^{1}$, Claudia S. Leeger-Aschmann ${ }^{1}$, Tanja H. Kakebeeke ${ }^{2,3}$, Annina E. Zysset ${ }^{2}$, Nadine Messerli-Bürgy ${ }^{4,5}$, Kerstin Stülb ${ }^{4}$, Amar Arhab ${ }^{5}$, Andrea H. Meyer ${ }^{6}$, Simone Munsch ${ }^{4+}$, Jardena J. Puder ${ }^{5,7 \dagger}$, Oskar G. Jenni ${ }^{2,3+}$ and Susi Kriemler ${ }^{1 *+}$
\end{abstract}

\begin{abstract}
${ }^{1}$ Epidemiology, Biostatistics and Prevention Institute, University of Zurich, Zurich, Switzerland, ${ }^{2}$ Child Development Center, University Children's Hospital Zurich, Zurich, Switzerland, ${ }^{3}$ Children's Research Center, University Children's Hospital Zurich, Zurich, Switzerland, ${ }^{4}$ Department of Clinical Psychology and Psychotherapy, University of Fribourg, Clinical Psychology and Psychotherapy, Fribourg, Switzerland, ${ }^{5}$ Endocrinology, Diabetes \& Metabolism Service, Centre Hospitalier Universitaire Vaudois (CHUV), Lausanne, Switzerland, ${ }^{6}$ Department of Psychology, University of Basel, Basel, Switzerland, ${ }^{7}$ Division of Pediatric Endocrinology, Diabetology and Obesity, Centre Hospitalier Universitaire Vaudois (CHUV), Lausanne, Switzerland
\end{abstract}

OPEN ACCESS

Edited by:

Tim Takken,

University Medical Center

Utrecht, Netherlands

Reviewed by:

Shahanawaz Syed,

University of Hail, Saudi Arabia

Eero Haapala,

University of Jyvaskyla, Finland

*Correspondence:

Susi Kriemler

susi.kriemlerwiget@uzh.ch

†These authors share last authorship

Specialty section:

This article was submitted to

Children and Health,

a section of the journal

Frontiers in Public Health

Received: 21 August 2018 Accepted: 06 February 2020 Published: 21 February 2020

Citation:

Schmutz EA, Leeger-Aschmann CS, Kakebeeke TH, Zysset AE, Messerli-Bürgy N, Stülb K, Arhab A,

Meyer AH, Munsch S, Puder JJ, Jenni OG and Kriemler S (2020) Motor Competence and Physical Activity in

Early Childhood: Stability and Relationship.

Front. Public Health 8:39.

doi: 10.3389/fpubh.2020.00039
Background: Normal motor development and adequate levels of physical activity engagement during the early years of life form the foundation of long-term psychological and physiological health. This is one of the very few studies that investigate the stability and relationships of motor competence and physical activity in preschool children.

Methods: Baseline and 12 month follow-up data of physical activity and motor competence of 550 preschool children aged 2-6 years from the Swiss Preschoolers' Health Study were used for this work. Physical activity data, expressed in counts per minute for total physical activity and minutes per day for time spent moderately-to-vigorously physically active, were collected over 1 week using accelerometers. Motor competence was assessed with the Zurich Neuromotor Assessment. Both motor competence and physical activity were age- and sex-adjusted. To examine the individual stability of physical activity and motor competence and reciprocal cross-sectional and longitudinal effects between these two domains, a latent variable cross-lagged panel model where motor competence was represented through a latent construct was examined using structural equation modeling.

Results: A weak cross-sectional correlation of motor competence with total physical activity $(r=0.24)$ and moderate-to-vigorous physical activity $(r=0.23)$ was found. Motor competence exhibited high stability $(\beta=0.82)$ in the preschool years and physical activity was moderately stable with estimates ranging from $\beta=0.37$ for total physical activity to $\beta=0.48$ for moderate-to-vigorous physical activity. In contrast to the autoregressive coefficients denoting individual stability, both cross-lagged effects were negligible indicating that physical activity was not a determinant of motor competence or vice versa.

Conclusions: Motor competence and physical activity developed independently of each other in early childhood. Although measures of quantity and intensity of physical activity were not related to motor development, specific movement experiences 
and practice-which are not reflected by accelerometry-may be needed for skill development. Future research should focus on examining what type of physical activity is important for motor development and how to assess it, and also whether the relationship between physical activity and motor competence evolves over time.

Clinical Trial Registration: Current Controlled Trials ISRCTN41045021 (date of registration: 21.03 .14$)$

Keywords: motor competence, fundamental movement skills, physical activity, children, preschool, longitudinal, splashy

\section{INTRODUCTION}

Both physical activity (PA) and motor competence (MC) have been linked to improved health indicators including increased cardiorespiratory fitness and decreased adiposity (1-3). MC is a global term used to describe goal-directed gross movements that involve large muscle groups or the whole body (e.g., running, jumping, balancing) (3). In early childhood, children begin to learn how to move their body through space by developing so called fundamental movement skills, which form the foundation for future more complex movement skills. Motor development is an iterative learning process driven by changes in the structure or function of the body as well as the environment $(4,5)$. There is increasing agreement about the existence of a continuous interplay of nature and nurture in defining motor development. Theories have moved away from neuro-maturational approaches claiming a predetermined sequence of motor skill acquisition (6) to a more holistic view involving contextual and biological factors $(5,7)$. From the dynamic systems perspective motor skills develop in a perpetual interplay between the organism, environment and task constraints, which may vary across stages of development. PA, an important element of this complex system, is a behavior that is needed to attain or improve MC. At the same time it can be seen as a product of motor development. This chicken-and-egg dilemma has been feeding the debate about how MC and PA are related over decades $(8,9)$.

Since evidence suggests that behavioral capabilities and lifestyle habits establish in childhood and track over time (10, 11), appropriate development of MC and levels of PA are important not only for child health, but also to sustain health throughout life. Numerous researchers have investigated underlying pathways including inter- and intra-individual variation and relationships. While cross-sectional evidence indicates a positive association between $\mathrm{MC}$ and PA in children and youth (12-14), only a few longitudinal studies investigating the suspected causal pathways between MC and PA have been published (15-19). Studies in older children found MC and

\footnotetext{
Abbreviations: BMI, body mass index; CFA, confirmatory factor analysis; CFI, comparative fit index; cpm, counts per minute; FIML, full information maximum likelihood; ISEI, international socio-economic index; MAR, missing at random; MC, motor competence; MCAR, missing completely at random; min, minute; MVPA, moderate-to-vigorous physical activity; PA, physical activity; RMSEA, rfoot mean square error of approximation; SD, standard deviation; SES, socioeconomic status; SRMR, standardized root mean square residual; TPA, total physical activity.
}

self-reported PA to be unrelated (20), MC to be predictive of subsequent self-reported PA $(16,17)$, objectively measured PA to be predictive of subsequent MC (21) or a reciprocal longitudinal relationship between MC and self-reported (18) or objectively measured (19) PA. The only study focusing specifically on preschool children showed that objectively measured moderateto-vigorous PA at 3.5 years, but not at 19 months, was predictive of locomotor skills at age 5 (15).

Thus, current evidence relies on predominantly crosssectional studies that show overall small effects. Whether a real causal pathway exists, and if so, whether it is unidirectional or reciprocal is unclear. To better understand how PA and MC are related in early childhood, more longitudinal studies that allow for cause-effect pathways in both directions are needed. The concept of reciprocal influence was first described in 2008 by Stodden et al. (8). The authors developed a theoretical framework where the direction of causation was hypothesized to change from early to middle childhood. In young children, PA was suggested to drive the development of MC through a variety of exploratory and structured movement experiences that promote neuromotor development. As children transition to middle and late childhood, the relationship was hypothesized to become stronger and more reciprocal, driven by the child's ability to perceive its competence in various movement contexts.

To the best of our knowledge, no longitudinal study has investigated the stability and reciprocal relationship of objectively measured PA and MC focusing specifically on preschool children. Such studies are important not only from a public health perspective, enabling the design of more effective and properly timed preventive measures or interventions that ultimately inform guidelines and recommendations. Also on the individual level, a thorough understanding of the stability and interplay can help predict performance and identify children with mild to severe delay or impairment of motor development requiring specialized clinical intervention during this crucial window of early childhood. Thus, to move research forward we investigated (a) the cross-sectional association between MC and PA, (b) the individual stability over time and (c) the longitudinal reciprocal relationship of MC and PA in preschool children. Because some evidence suggests that besides quantity of PA the intensity may affect the relationship between PA and MC, we used two different constructs of PA [total PA (TPA) and moderate-to-vigorous PA (MVPA)] (2). We also examined a potential moderating effect of sex and age group (younger vs. older) on the aforementioned research questions. 


\section{MATERIALS AND METHODS}

\section{Study Population}

Data presented in this work are drawn from the Swiss Preschoolers' Health Study (SPLASHY; ISRCTN41045021), a multi-site prospective cohort study including 5552 - to 6 yearold preschool-aged children from 84 childcare centers located in five cantons of Switzerland (covering 50\% of the Swiss population in 2013). Sampling of childcare centers was stratified according to one stratum with four levels: urban community and rural community with high socio-economic status (SES; above-average) and low SES (below-average), each based on the prevalence of child care centers in the respective communities. In total, 639 child care centers were contacted between January 2013 and October 2014, of which 126 child care centers agreed to participate and to inform the parents. Forty-two centers were excluded after the preparation of testing dates due to too few (less than two) participating children $(78 \%)$ or for other reasons (12\%). Data collection in childcare centers was conducted in 2014 and 1 year later by the same study team in parallel at all study sites. Children recruited in $2014(n=476)$ had a followup assessment 1 year later. Those recruited in $2015(n=79)$ had a baseline assessment only. Both baseline (T0) and followup (T1) data are used in the current study. Ethical approval is in accordance with the Declaration of Helsinki and has been obtained from all local ethical committees (No 338/13 for the Ethical Committee of the Canton of Vaud as the main approving authority). Children and parents provided oral and written informed consent. A detailed description of the study design has been published elsewhere (22).

\section{Measures}

\section{Physical Activity}

PA was objectively monitored on seven consecutive days using a hip-worn accelerometer (wGT3X-BT, ActiGraph, Pensacola, FL, USA). Participants were instructed to wear the monitor 24 $\mathrm{h}$ /day except during water-based activities. A sampling frequency of $30 \mathrm{~Hz}$ was used. Accelerometer data were downloaded in 3-s epochs and aggregated to $15 \mathrm{~s}$ epochs. Nighttime hours ( $9 \mathrm{pm}$ to $7 \mathrm{am}$ ) and non-wear periods, defined as $\geq 20 \mathrm{~min}$ of consecutive zero counts on all axes (23), were excluded. A monitoring day was considered valid if at least $10 \mathrm{~h}$ of activity were recorded. PA outcome data were expressed as counts per minute [cpm] for TPA and min/day for MVPA (defined as $\geq 420$ counts per $15 \mathrm{~s}$ ) (24). Since at both time points TPA and MVPA did not differ between participants who provided at least 3 valid days including 1 weekend day (baseline: $91 \%$, follow-up: $89 \%$ ), and those with less days of recording, all participants were included in analysis.

\section{Motor Competence}

After measuring height and body weight by standard procedures, MC was assessed using the Zurich Neuromotor Assessment 35 (ZNA 3-5) $(25,26)$, which is based on the original ZNA for children older than 5 years (ZNA 5-18) $(27,28)$. The ZNA 3-5 is a well-standardized motor test instrument with good intra-observer (0.56-1.00) and inter-observer (0.42-0.99) reliability, while test-retest reliability is lower in some tasks (0.350.84) (25). Five components were used to capture gross motor proficiency: static balance, walking on a straight line, sideward jumping, hopping on one leg and running. All tasks were videotaped, which allowed offline rating. The examiner explained and demonstrated each task. If children did not understand the task or did something different, the demonstration was repeated. In case of a second failure, the examiner scored the task as "failed" and continued the assessment. Instructions for the tasks were as follows: (1) Static balance: Children were asked to do a one-leg stand for as long as possible. Time counting started as soon as the child lifted one foot off the floor and stopped when the child touched the floor with the lifted foot or shifted the foot of the standing leg more than $2 \mathrm{~cm}$. The same procedure was repeated for the other leg. A qualitative score from 0 to 4 was given: 0 $=$ one-leg stand more than $5 \mathrm{~s}$ on both legs; $1=$ one-leg stand more than 5 s on only one leg; $2=$ one-leg stand between 2 and $5 \mathrm{~s}$ on both legs; $3=$ one-leg stand between 2 and $5 \mathrm{~s}$ on only one leg; $4=$ not able to stand on either leg for more than $2 \mathrm{~s}$. (2) Walking on a straight line: Children were asked to walk on a straight line consisting of an elastic band placed on the floor putting one foot in front of the other such that the heel of the front foot touched the toes of the back one. Rating included a qualitative score from 0 to 4 : 0 = perfect performance, heel touches toes; $1=$ feet straight on the line but gap between the feet; 2 = feet not straight and/or off the line up to 3 times; $3=$ feet perpendicular and/or feet off the line more than 3 times; 4 $=$ not able to walk on the line. (3) Jumping sideways: Children were asked to jump sideways over the elastic band back and forth keeping the feet together. Rating included a qualitative score from 0 to 4 : $0=$ perfect performance, very smooth jumping; $1=$ jumping performed correctly but not very smoothly; $2=$ touchdown with both feet at the same time, stiff movements; $3=$ total body involvement, poor coordination in relation to the band direction; 4 = Jumping over the elastic band but not in relation to the band direction. (4) Hopping on one leg: Children were asked to hop as many times as possible on one leg. Two trials per leg were given. Rating included a qualitative score from 0 to 4 : $0=$ hopping on both legs more than 7 times; 1 = hopping on only one leg more than 3 times; 2 = hopping on both legs up to 3 times; 3 = hopping on only one leg 1-3 times; $4=$ cannot hop on either leg. (5) Running: Children were asked to run around the cord (at least 20 meters). Rating included a qualitative score from 0 to 4 : $0=$ rolling motion of feet with adjustment of upper body; $1=$ rolling motion of feet, stiff upper body; 2 = running with partial rolling motion of feet; 3 = running without rolling motion of feet; $4=$ cannot run (no flight phase).

\section{Statistical Analyses}

Statistical analyses were performed using $\mathrm{R}$ version 3.4.4 ( $\mathrm{R}$ Foundation for Statistical Computing, Vienna, Austria). Descriptive statistics are presented as mean [standard deviation [SD]] and ranges for continuous variables and percentages for categorical variables, unless stated otherwise. Participants without PA and MC data were excluded from the analysis $(n=5)$, resulting in a sample of 550 individuals. Q-Q plots and frequency distributions were used to check for normal distribution and 
potential outliers. MC measures were standardized and expressed as standard deviation scores calculated from age- and sexadjusted normative values to receive identical metrics across tasks. PA was also age- and sex-standardized to account for known age and sex effects. Thus, positive values correspond to above average performance or PA, respectively, and negative values indicate below average measurements within the same sex and age group. To investigate the hypothesized reciprocal longitudinal relationship between $\mathrm{MC}$ and $\mathrm{PA}$, a latent variable cross-lagged panel model using structural equation modeling was created, where MC was represented through a latent construct (29). The latent constructs were first verified as separate measurement models with confirmatory factor analysis (CFA). The CFA and latent variable cross-lagged panel model were performed using the package lavaan (30). Full information maximum likelihood (FIML) was used to handle missing data and results were compared to a complete case analysis ( $n$ $=218$ ). FIML is known to lead to unbiased estimates if the data are either missing completely at random (MCAR) or missing at random (MAR) whereas complete case analysis requires MCAR for unbiased estimates and suffers from reduced power due to reduction in sample size. In line with previous recommendations, good model-data fit was characterized by a non-significant $\chi 2$-test statistic, a standardized root mean square residual (SRMR) $<0.08$, a root mean square error of approximation (RMSEA) $<0.06$, and a comparative fit index (CFI) $>0.90$ (31). Bootstrapping was used to ensure robustness of model fit indices (500 bootstrap replications) $(32,33)$. Estimated paths were adjusted for accelerometer wear time. Additional analyses were conducted to further evaluate the robustness of our findings: (1) Multigroup structural equation modeling was applied to investigate whether sex or age group (younger vs. older children by using the sample median) moderated the relationship between $\mathrm{PA}$ and $\mathrm{MC}$, (2) Estimated paths were additionally controlled for SES and excess body weight to exclude potential confounding. SES was assessed using the International Socio-Economic Index of occupational status (ISEI) $(34,35)$, which assigns values between 16 (manual labor in agricultural sector) and 90 (judge) to job titles with respect to education and income. BMI z-scores, calculated based on the World Health Organization growth charts were used as an indicator for body fatness. The statistical significance level alpha was set at 0.05 .

\section{RESULTS}

Descriptive statistics are shown in Table 1. The sample comprised 550 preschool children (47\% boys) aged $3.9(0.7)$ years at baseline and 4.9 (0.7) at follow-up. Comparisons between baseline and follow-up measurements demonstrated that on average children performed better in MC tests and were more physically active at follow-up (all $p<0.05$, paired $t$-tests). All children met the guidelines of at least $180 \mathrm{~min}$ of any PA per day at both time points (37-39). There were no differences in baseline demographic characteristics (age, SES, BMI) between children with complete and incomplete data (data not shown). Both MC measurement models (baseline and follow-up) showed a good fit;
TABLE 1 | Characteristics of participants at baseline and follow-up $(n=550)$.

\begin{tabular}{|c|c|c|c|c|}
\hline & \multicolumn{2}{|c|}{ Baseline } & \multicolumn{2}{|c|}{ Follow-up } \\
\hline & Mean (SD) & Range & Mean (SD) & Range \\
\hline Age (years) & $3.9(0.7)$ & $2.2-6.6$ & $4.9(0.7)$ & $3.2-7.6$ \\
\hline BMl z-score ${ }^{a}$ & $0.4(1.0)$ & $-4.0-4.7$ & $0.3(0.9)$ & $-3.3-5.4$ \\
\hline SES & $62.9(15.5)$ & $17.0-89.0$ & $62.5(15.5)$ & $17.0-89.0$ \\
\hline Monitor wear time [h/day] & $12.8(0.7)$ & $10.2-14.0$ & $13.0(0.9)$ & $10.2-14.0$ \\
\hline TPA [cpm] & $623(153)$ & $243-1,331$ & $643(154)$ & $260-1,797$ \\
\hline MVPA [min/day] & $92(29)$ & 26-206 & $97(28)$ & 19-201 \\
\hline Walking ${ }^{b}$ & $2.2(0.8)$ & $0-4$ & $1.8(0.8)$ & $0-4$ \\
\hline Jumping ${ }^{b}$ & $2.6(1.0)$ & $0-4$ & $2.0(1.2)$ & $0-4$ \\
\hline Hopping ${ }^{b}$ & $2.4(1.4)$ & $0-4$ & $1.2(1.3)$ & $0-4$ \\
\hline Running ${ }^{b}$ & $2.4(0.8)$ & $0-4$ & $1.9(0.6)$ & $0-4$ \\
\hline Static balance ${ }^{c}[\mathrm{sec}]$ & $8.0(8.0)$ & $2-75$ & $12.4(10.4)$ & $2-88$ \\
\hline
\end{tabular}

a Based on WHO growth standards (36). ${ }^{b}$ Measured on an ordinal scale, lower scores indicate better performance; ${ }^{C}$ based on dominant leg, longer duration indicates better performance; BMI, Body mass index; SES, Socioeconomic status; TPA, Total physical activity; cpm, counts per minute; MVPA, moderate-to-vigorous physical activity.

$\chi 2(5)=0.59, p=0.99 ; \mathrm{SRMR}=0.01 ; \mathrm{RMSEA}=0.00 ; \mathrm{CFI}=1.00$ and $\chi 2(5)=1.16, p=0.76$; SRMR $=0.01$; RMSEA $=0.00$; CFI $=1.00$. Similarly, the latent variable cross-lagged panel models with TPA $[\chi 2(45)=41.05, p=0.47$; SRMR $=0.02$; $\mathrm{RMSEA}=$ 0.00 ; CFI $=0.99]$ and MVPA $[\chi 2(45)=39.11, p=0.46$; SRMR $=0.03$; RMSEA $=0.00$; CFI $=0.99$ ] demonstrated good overall fit. No post-hoc modifications were conducted.

Figure 1 depicts the latent variable cross-lagged panel models for TPA (A) and MVPA (B), respectively, for the whole group. $\mathrm{MC}$ was highly stable over time with an autoregressive coefficient of $\beta=0.82(p<0.001)$. Both TPA and MVPA showed moderate stability, autoregressive coefficients were $\beta=0.37(p<0.001)$ and $\beta=0.48(p<0.001)$, respectively. Cross-lagged coefficients between PA and MC were very small and not significant [standardized coefficients for the effect of TPA at baseline on MC at follow-up: $\beta=0.02(p=0.77)$, for the effect of MC at baseline on TPA at follow-up $\beta=0.10(p=0.25)]$. A significant but weak cross-sectional association of MC with TPA and MVPA was found at baseline $[r=0.24(p<0.001)$ and $r=0.23(p=$ $0.001)$, respectively]. Multigroup analyses revealed that neither age group nor sex had a moderating effect on the relationships between MC and PA in the model. Furthermore, adjusting for SES and BMI z-score did not change the observed effects.

\section{DISCUSSION}

To the best of our knowledge, this is the first study to examine both the stability and reciprocal relationship of $\mathrm{MC}$ and PA within a relatively large cohort of preschoolers using objective measures. We found that at this young age, children demonstrated diverse levels of MC and PA that were unrelated. Individual stability, i.e., the tendency to maintain the same relative position within a cohort, was relatively high for MC and moderate for PA over a 1 year period. Our findings suggest that 


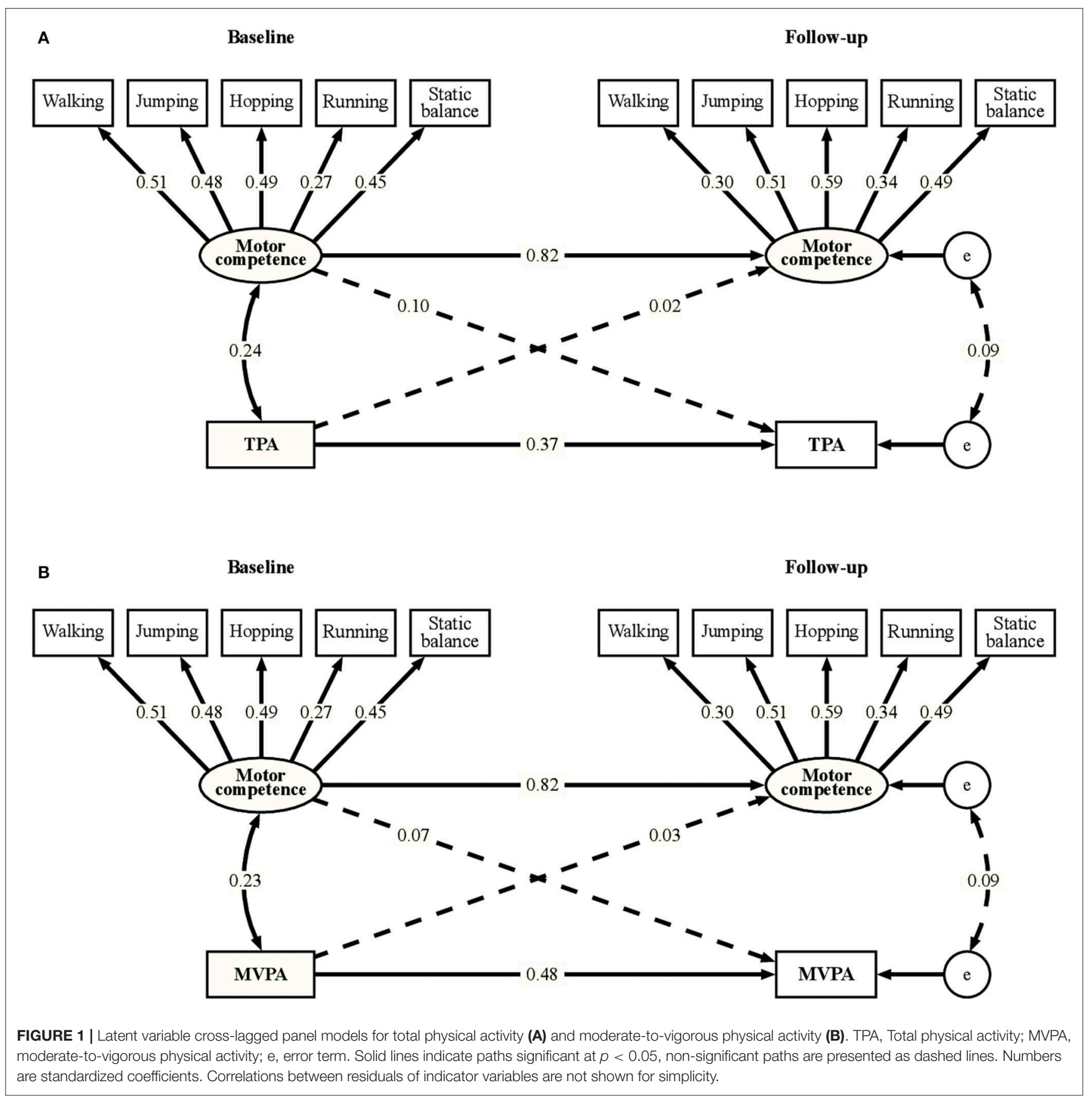

MC and PA develop independently of each other and track over time during the period of early childhood.

Although we found a significant but weak cross-sectional association between PA and MC, analysis of cross-lagged effects indicated that PA was not predictive of MC in early childhood or vice versa. Previous cross-sectional studies in preschoolers found similar results $(21,40,41)$. A study of 394 3-5 yearold children (40) reported slightly lower but significant positive correlations of TPA $(r=0.10)$ and percent time in MVPA $(r=0.18)$ with total movement skills. Others found similar correlations with total movement skills (MVPA, $r=0.20$; VPA, $r=0.26$ ) (41) and dynamic balance skills (TPA, $r=$ 0.20 ; MVPA, $r=0.22$ ) (21). The only study examining the longitudinal relationship in young children $(n=185)$ found that MVPA at 3.5 years predicted locomotor skills, but not object control skills or total skill competence, at age 5 (15). Investigations in older children reported mixed results (1620), which further illustrates that the nature and strength of the relationship may not be straightforward. A possible explanation for our null result is the way through which 
PA was operationalized. Although habitual PA per se may be important for some health-related outcomes, it may not promote MC. Specific types or quality of movement experiences may be required rather than overall movement quantity or intensity. High participation in balancing activities for example would not be represented by high levels of total or moderateto-vigorous PA, yet would likely be associated with better balancing competence. This idea has previously been confirmed by a study investigating participation in different physical education activities in preschool aged children (42). Similarly, the implementation of planned movement programs was found to be effective at improving MC as compared to "free play" $(43,44)$. Along the same lines, it is important to consider how MC has been operationalized when analyzing the relationship with PA and comparing results. A systematic review of motor skill correlates found that only some aspects of MC were related to PA (14). While skill composite and motor coordination had a positive association with PA, evidence was indeterminate for object control and locomotor skills. Lastly, the use of different PA assessment tools (self-report, direct observation, or accelerometry) and $\mathrm{MC}$ instruments (quantitative vs. qualitative assessment batteries) as well as analysis approaches of PA [choice of epoch length and intensity cut-points (45)] can greatly impact the results.

We further hypothesized that the relationship between MC and PA strengthens as children age. As children transition to middle childhood, the sum of all influences is thought to lead to a positive or negative spiral of engagement that compounds over time (8) and affects health-related risk factors. Individuals with low actual and perceived MC for example will be drawn into a negative spiral of disengagement resulting in reduced sport involvement, low levels of PA or even obesity (8). While $70 \%$ of the children in our sample were below the age of 4 at baseline and potentially primarily constrained by endogenous developmental steps of maturation (14), we hypothesized that additional physiological or psychological factors, such as children's perceptions of their own MC, may develop at 5-6 years and influence the relationship. Yet, we did not find the relationship to strengthen as children aged. We assume that a longer time span beyond the fundamental movement skills development period would be required to capture the expected increase in strength of association (8).

Our findings indicated that $\mathrm{MC}$ was stable over time during the preschool period whereas PA exhibited moderate stability. Somewhat lower stability coefficients were found for MC in a longitudinal study from 4 to 5 and 6 years (boys: 0.58-0.69, girls: $0.31-0.47 ; N=205$ ) (11) and similar coefficients were found for PA $(10,46)$. It is plausible that the lower stability coefficients of PA compared to MC in early childhood are due to different etiological pathways and adaptability to internal and external factors. Habitual PA as a behavior may entail more flexibility and thus greater variation than a construct that at this stage is, at least in part, influenced by biological maturation (7).

Previous literature has not offered a clear answer as to whether the stability and relationship of PA and MC differed by sex
(13). Some authors found different developmental trajectories between the two sexes, particularly in later childhood and adolescence, such that girls were more proficient in locomotion components whereas boys scored better in object control tasks (47-50). The MC assessment battery we used did not assess object control/manipulation skills, which could be a possible explanation of why we did not find a moderating effect of sex. However, this difference has often been found in middle childhood and adolescence and basic patterns were shown to be similar in boys and girls during the preschool period $(11,14)$. This suggests that various opportunities for practice may impact the development of specific skills (14, 43) and that opportunities and motivation for engagement in different context- or skill-specific activities may vary for boys and girls.

Important strengths of this study include (a) the longitudinal panel design, which allowed drawing some conclusions on change over time and direction of association, (b) the relatively large cohort of children from 2 to 6 years covering the whole preschool range, (c) the fact that PA was objectively assessed and MC was videotaped and rated offline by experts and (d) the use of state-of-the-art statistical methods to model latent constructs and test complex reciprocal relationships. Limitations that need to be addressed when interpreting our results include the short follow up time, the fact that not the entire range of motor skills was assessed (e.g., no object control) and that accelerometers likely underestimate PA in preschoolers as waterbased and rolling activities are insufficiently reflected (23). Another potential weakness is that MVPA was defined using fixed cut-offs, which has a high probability of misclassification of MVPA because individual variation in such cut-offs. We are confident that taking another cutoff for MVPA would not change the result of the paper as we have also presented data with similar results on total PA that provides a safer way. Although we do not know whether children who do not attend childcare behave differently, the generalizability of our findings may be limited by the fact that our sample included only children who attended childcare at least twice a week.

This is the first longitudinal study that provides evidence on the stability and reciprocal relationship of PA and MC in young children. Based on our findings MC is a stable construct that is not influenced by the quantity or intensity of habitual PA at this early age. To inform the design of effective interventions, future longitudinal studies should examine what type of physical activity is important for motor skill development, how to assess it, and whether the (reciprocal) relationship between PA and MC evolves as children age.

\section{ETHICS STATEMENT}

Ethical approval is in accordance with the Declaration of Helsinki and has been obtained from all local ethical committees (No 338/13 for the Ethical Committee of the Canton of Vaud as the main approving authority). Children and parents provided oral and written informed consent. 


\section{AUTHOR CONTRIBUTIONS}

Conceived and designed the Splashy study: JP, SK, SM, and OJ. Performed data collection: ES, CL-A, AZ, TK, KS, NM-B, and AA. Designed the research, had full access to all data, and take responsibility for the integrity of data and accuracy of data analysis: ES and SK. Assisted in statistical data analysis: AM. Performed data analysis and wrote, reviewed, and edited the manuscript: ES. All authors reviewed, edited, and approved the manuscript.

\section{REFERENCES}

1. Timmons BW, Leblanc AG, Carson V, Connor Gorber S, Dillman C, Janssen I, et al. Systematic review of physical activity and health in the early years (aged 0-4 years). Appl Physiol Nutr Metab. (2012) 37:77392. doi: 10.1139/h2012-070

2. Lubans DR, Morgan PJ, Cliff DP, Barnett LM, Okely AD. Fundamental movement skills in children and adolescents: review of associated health benefits. Sports Med. (2010) 40:101935. doi: 10.2165/11536850-000000000-00000

3. Robinson LE, Stodden DF, Barnett LM, Lopes VP, Logan SW, Rodrigues LP, et al. Motor competence and its effect on positive developmental trajectories of health. Sports Med. (2015) 45:1273-84. doi: 10.1007/s40279-015-0351-6

4. Clark JE. On becoming skillful: patterns and constraints. Res Q Exerc Sport. (1995) 66:173-83. doi: 10.1080/02701367.1995.10608831

5. Newell KM. Constraints in the development of coordination. In: Wade MG, Whiting HTA, editors. Motor Development in Children: Aspects of Coordination and Control. Dordrecht: The Netherlands Martinus Nijhoff Publishers (1986). p. 341-60. doi: 10.1007/978-94-009-4460-2_19

6. Salkind NJ. Theories of Human Development. New York, NY: Van Nostrand Reinhold (1981).

7. Metcalfe J, Clark JE. The mountain of motor development: a metaphor. In: Clark JE, Humphrey JH, editors. Motor Development: Research and Reviews. Reston, VA: NASPE Pulications (2002). p. 163-90.

8. Stodden DF, Goodway JD, Langendorfer SJ, Roberton MA, Rudisill ME, Garcia C, et al. A developmental perspective on the role of motor skill competence in physical activity: An emergent relationship. Quest. (2008) 60:290-306. doi: 10.1080/00336297.2008.10483582

9. Seefeldt V. Developmental motor patterns: implications for elementary school physical education. In: Nadeau C, Holliwell W, Newell K, Roberts G, editors. Psychology of Motor Behavior and Sport. Champaign, IL: Human Kinetics (1980). p. 314-23.

10. Jones RA, Hinkley T, Okely AD, Salmon J. Tracking physical activity and sedentary behavior in childhood: a systematic review. Am J Prev Med. (2013) 44:651-8. doi: 10.1016/j.amepre.2013.03.001

11. Ahnert J, Schneider W, Bös K. Developmental changes and individual stability of motor abilities from the preschool period to young adulthood. In: Schneider W, Bullock M, editors. Human Development From Early Childhood to Early Adulthood. New York, NY: Psychology Press (2009). p. 35-62. doi: 10.4324/9780203888544

12. Figueroa R, An R. Motor skill competence and physical activity in preschoolers: a review. Matern Child Health J. (2017) 21:136-46. doi: 10.1007/s10995-016-2102-1

13. Holfelder B, Schott N. Relationship of fundamental movement skills and physical activity in children and adolescents: a systematic review. Psychol Sport Exerc. (2014) 15:382-91. doi: 10.1016/j.psychsport.2014.03.005

14. Barnett LM, Lai SK, Veldman SLC, Hardy LL, Cliff DP, Morgan PJ, et al. Correlates of gross motor competence in children and adolescents: a systematic review and meta-analysis. Sports Med. (2016) 46:166388. doi: 10.1007/s40279-016-0495-Z

15. Barnett LM, Salmon J, Hesketh KD. More active pre-school children have better motor competence at school starting age: an observational cohort study. BMC Public Health. (2016) 16:1068. doi: 10.1186/s12889-016-3742-1

\section{FUNDING}

This study was funded by the Swiss National Science Foundation (CRSII3_147673) and the Jacobs Foundation.

\section{ACKNOWLEDGMENTS}

We thank the children and parents who gave us their time and a team of dedicated study staff for their assistance in data collection.

16. Lopes VP, Rodrigues LP, Maia JA, Malina RM. Motor coordination as predictor of physical activity in childhood. Scand J Med Sci Sports. (2011) 21:663-9. doi: 10.1111/j.1600-0838.2009.01027.x

17. Barnett LM, van Beurden E, Morgan PJ, Brooks LO, Beard JR. Childhood motor skill proficiency as a predictor of adolescent physical activity. J Adolesc Health. (2009) 44:252-9. doi: 10.1016/j.jadohealth.2008.07.004

18. Jaakkola $\mathrm{T}$, Washington $\mathrm{T}$. The relationship between fundamental movement skills and self-reported physical activity during Finnish junior high school. Phys Educ Sport Pedagogy. (2013) 18:492-505. doi: 10.1080/17408989.2012.690386

19. Lima RA, Pfeiffer K, Larsen LR, Bugge A, Moller NC, Anderson LB, et al. Physical activity and motor competence present a positive reciprocal longitudinal relationship across childhood and early adolescence. J Phys Act Health. (2017) 14:440-7. doi: 10.1123/jpah.2016-0473

20. dos Santos MAM, Nevill AM, Buranarugsa R, Pereira S, Gomes TNQF, Reyes A, et al. Modeling children's development in gross motor coordination reveals key modifiable determinants. An allometric approach. Scand J Med Sci Sports. (2018) 28:1594-603. doi: 10.1111/sms.13061

21. Burgi F, Meyer U, Granacher U, Schindler C, Marques-Vidal P, Kriemler $\mathrm{S}$, et al. Relationship of physical activity with motor skills, aerobic fitness and body fat in preschool children: a cross-sectional and longitudinal study (Ballabeina). Int J Obes. (2011) 35:937-44. doi: 10.1038/ijo.2011.54

22. Messerli-Burgy N, Kakebeeke TH, Arhab A, Stulb K, Zysset AE, LeegerAschmann CS, et al. The Swiss Preschoolers' health study (SPLASHY): objectives and design of a prospective multi-site cohort study assessing psychological and physiological health in young children. BMC Pediatr. (2016) 16:85. doi: 10.1186/s12887-016-0617-7

23. Cliff DP, Reilly JJ, Okely AD. Methodological considerations in using accelerometers to assess habitual physical activity in children aged $0-5$ years. $J$ Sci Med Sport. (2009) 12:557-67. doi: 10.1016/j.jsams.2008.10.008

24. Pate RR, Almeida MJ, McIver KL, Pfeiffer KA, Dowda M. Validation and calibration of an accelerometer in preschool children. Obesity. (2006) 14:2000-6. doi: 10.1038/oby.2006.234

25. Kakebeeke TH, Caflisch J, Chaouch A, Rousson V, Largo RH, Jenni OG. Neuromotor development in children. Part 3: motor performance in 3- to 5year-olds. Dev Med Child Neurol. (2013) 55:248-56. doi: 10.1111/dmcn.12034

26. Kakebeeke TH, Locatelli I, Rousson V, Caflisch J, Jenni OG. Improvement in gross motor performance between 3 and 5 years of age. Percept Mot Skills. (2012) 114:795-806. doi: 10.2466/10.13.25.PMS.114.3.795-806

27. Largo RH, Caflisch JA, Hug F, Muggli K, Molnar AA, Molinari L, et al. Neuromotor development from 5 to 18 years. Part 1: timed performance. Dev Med Child Neurol. (2001) 43:436-43. doi: 10.1017/S0012162201000810

28. Largo RH, Caflisch JA, Hug F, Muggli K, Molnar AA, Molinari L. Neuromotor development from 5 to 18 years. Part 2: associated movements. Dev Med Child Neurol. (2001) 43:444-53. doi: 10.1017/S0012162201000822

29. Newsom JT. Longitudinal Structural Equation Modeling: A Comprehensive Introduction. New York, NY: Taylor \& Francis (2015). doi: 10.4324/9781315871318

30. Rosseel Y. lavaan: an R Package for structural equation modeling. J Stat Softw. (2012) 48:1-36. doi: 10.18637/jss.v048.i02

31. Lei $\mathrm{PW}, \mathrm{Wu} \mathrm{Q}$. Introduction to structural equation modeling: issues and practical considerations. Educ Meas. (2007) 26:3343. doi: 10.1111/j.1745-3992.2007.00099.x 
32. Bollen KA, Stine RA. Bootstrapping Goodness-of-Fit measures in structural equation models. Sociol Methods Res. (1992) 21:205-29. doi: 10.1177/0049124192021002004

33. Savalei V, Yuan KH. On the model-based bootstrap with missing data: obtaining a P-Value for a test of exact fit. Multivariate Behav Res. (2009) 44:741-63. doi: 10.1080/00273170903333590

34. Ganzeboom HBG. A new international socio-economic index [ISEI] of occupational status for the International Standard Slassification of Occupation 2008 [ISCO-08] constructed with data from the ISSP 2002-2007; with an analysis of quality of occupational measurement in ISSP. Annual Conference of International Social Survey Programme. Lisbon (2010).

35. Ganzeboom HBG, De Graaf PM, Treiman DJ. A standard international socio-economic index of occupational status. Soc Sci Res. (1992) 21:156. doi: 10.1016/0049-089X(92)90017-B

36. WHO Multicentre Growth Reference Study Group. WHO Child Growth Standards: Length/Height-for-Age, Weight-for-Age, Weight-for-Length, Weight-for-Height and Body Mass Index-for-Age: Methods and Development. Geneva: World Health Organization (2006).

37. Canadian Society for Exercise Physiology. Canadian Physical Activity Guidelines. Ottawa, ON (2012).

38. Department of Health, Physical Activity, Health Improvement and Protection. Start Active, Stay Active: a report on physical activity from the four home countries' Chief Medical Officers. London: Department of Health, Physical Activity, Health Improvement and Protection (2011).

39. Department of Health and Aging, Australia. Get Up and Grow: Healthy Eating and Physical Activity for Early Childhood. Canberra, ACT: Commonwealth of Australia (2009).

40. Fisher A, Reilly JJ, Kelly LA, Montgomery C, Williamson A, Paton JY, et al. Fundamental movement skills and habitual physical activity in young children. Med Sci Sport Exerc. (2005) 37:684-8. doi: 10.1249/01.MSS.0000159138.48107.7D

41. Williams HG, Pfeiffer KA, O’Neill JR, Dowda M, McIver KL, Brown WH, et al. Motor skill performance and physical activity in preschool children. Obesity. (2008) 16:1421-6. doi: 10.1038/oby.2008.214

42. Barnett L, Hinkley T, Okely AD, Salmon J. Child, family and environmental correlates of children's motor skill proficiency. J Sci Med Sport. (2013) 16:3326. doi: 10.1016/j.jsams.2012.08.011

43. Logan SW, Robinson LE, Wilson AE, Lucas WA. Getting the fundamentals of movement: a meta-analysis of the effectiveness of motor skill interventions in children. Child Care Health Dev. (2012) 38:305-15. doi: 10.1111/j.1365-2214.2011.01307.x

44. Wick K, Leeger-Aschmann CS, Monn ND, Radtke T, Ott LV, Rebholz CE, et al. Interventions to promote fundamental movement skills in childcare and kindergarten: a systematic review and meta-analysis. Sports Med. (2017) 47:2045-68. doi: 10.1007/s40279-017-0723-1

45. Bornstein DB, Beets MW, Byun W, McIver K. Accelerometer-derived physical activity levels of preschoolers: a meta-analysis. J Sci Med Sport. (2011) 14:50411. doi: 10.1016/j.jsams.2011.05.007

46. Caldwell HA, Proudfoot NA, King-Dowling S, Di Cristofaro NA, Cairney J, Timmons BW. Tracking of physical activity and fitness during the early years. Appl Physiol Nutr Metab. (2016) 41:504-10. doi: 10.1139/apnm-2015-0338

47. McKenzie TL, Sallis JF, Broyles SL, Zive MM, Nader PR, Berry CC, et al. Childhood movement skills: predictors of physical activity in Anglo American and Mexican American adolescents? Res Q Exerc Sport. (2002) 73:23844. doi: 10.1080/02701367.2002.10609017

48. Butterfield SA, Loovis EM. Influence of age, sex, balance, and sport participation on development of throwing by children in grades K-8. Percept Mot Skills. (1993) 76:459-64. doi: 10.2466/pms.1993.76.2.459

49. Iivonen S, Sääkslahti AK. Preschool children's fundamental motor skills: a review of significant determinants. Early Child Dev Care. (2014) 184:110726. doi: 10.1080/03004430.2013.837897

50. Antunes AM, Maia JA, Gouveia ER, Thomis MA, Lefevre JA, Teixeira AQ, et al. Change, stability and prediction of gross motor co-ordination in Portuguese children. Ann Hum Biol. (2016) 43:201-11. doi: 10.3109/03014460.2015.1058419

Conflict of Interest: The authors declare that the research was conducted in the absence of any commercial or financial relationships that could be construed as a potential conflict of interest.

Copyright (C) 2020 Schmutz, Leeger-Aschmann, Kakebeeke, Zysset, Messerli-Bürgy, Stülb, Arhab, Meyer, Munsch, Puder, Jenni and Kriemler. This is an open-access article distributed under the terms of the Creative Commons Attribution License (CC $B Y)$. The use, distribution or reproduction in other forums is permitted, provided the original author(s) and the copyright owner(s) are credited and that the original publication in this journal is cited, in accordance with accepted academic practice. No use, distribution or reproduction is permitted which does not comply with these terms. 